\title{
Serum Dickkopf-1 levels as a clinical and prognostic factor in patients with bladder cancer
}

\author{
D.K. Sun, L. Wang, J.M. Wang and P. Zhang \\ Department of Urology, Yantai Yuhuangding Hospital, Yantai, Shandong, China \\ Corresponding author: P. Zhang \\ E-mail: yhd_zhangpeng@126.com \\ Genet. Mol. Res. 14 (4): 18181-18187 (2015) \\ Received August 28, 2015 \\ Accepted October 11, 2015 \\ Published December 23, 2015 \\ DOI http://dx.doi.org/10.4238/2015.December.23.5
}

ABSTRACT. Dickkopf-1 (DKK-1) is a secreted protein that inhibits Wnt signaling. However, the clinical significance and prognostic value of serum DKK-1 levels have not been previously investigated in bladder cancer in Chinese patients. Blood samples were taken from 94 consecutive patients diagnosed with bladder cancer and 60 healthy control subjects. Serum DKK-1 expression levels were measured by enzyme-linked immunosorbent assay according to the manufacturer's directions. The Kaplan-Meier method was used to estimate survival, and the log-rank test was used to test differences between the survival curves. Multivariate survival analysis was performed for all parameters deemed significant in the univariate analyses using the Cox regression model. The mean serum DKK-1 level in patients with bladder cancer was $35.91 \pm 16.09 \mathrm{ng} / \mathrm{mL}$, which was significantly higher than that in healthy individuals $(9.08 \pm 5.21 \mathrm{ng} / \mathrm{mL}, P$ $<0.001)$. Furthermore, serum DKK-1 levels were correlated significantly with lymph node metastasis $(P=0.021)$, distant metastasis $(P=0.013)$, and TNM stage $(P=0.008)$. Kaplan-Meier analysis using the log-rank test indicated that high serum DKK-1 levels were linked to poorer survival (33.4 vs $70.1 \% ; P=0.007)$. Multivariate analysis revealed that serum DKK-1 levels represented an independent prognostic factor for overall survival 
(hazard ratio $=2.365,95 \%$ confidence interval $=1.873-8.881, \mathrm{P}=0.029$ ). High serum DKK-1 levels may be associated with tumor progression and poor prognosis in bladder cancer and may be used as a potential biomarker to predict the prognosis of patients with bladder cancer.

Key words: Dickkopf-1; Bladder cancer; Prognosis; Biomarker

\section{INTRODUCTION}

Bladder cancer is the fourth most prevalent malignancy in men worldwide, and an estimated 430,000 patients are diagnosed with bladder cancer annually (Siegel et al., 2014). At the initial presentation, approximately $75 \%$ of patients have non-muscle-invasive (carcinoma in situ, Ta and T1) disease, whereas the remaining patients have disease that invades the muscularis propria (Ro et al., 1992). Although the TNM staging of bladder cancer guides both treatment and prognosis, there remains substantial heterogeneity among similarly staged patients with respect to treatment response and overall outcomes. Therefore, there is a critical need for the identification of biomarkers to diagnose bladder cancer at an early stage, monitor recurrence, refine prognostic estimates, and predict response to treatment in patients with bladder cancer.

Dickkopf-1 (DKK-1) is a secreted protein that inhibits the Wnt signaling pathway (Nusse, 2005). The expression and roles of DKK-1 differ in various cancers, and recent studies reported that overexpression of DKK-1 is found in many malignant tumors including breast cancer, lung cancer, esophageal carcinoma, and hepatocellular carcinoma (HCC), indicating a potential oncogenic function of DKK-1 (Patil et al., 2005; Forget et al., 2007; Yamabuki et al., 2007; Darlavoix et al., 2009; Makino et al., 2009). Previously, Kaba et al. (2014) investigated the clinical significance of serum DKK-1 levels in Turkish patients with bladder cancer, finding that serum DKK-1 levels were correlated with both disease progression and the tumor grade. However, the clinical significance and prognostic value of serum DKK-1 levels have not been previously investigated in bladder cancer in the Chinese population.

\section{MATERIAL AND METHODS}

\section{Patients and serum samples}

The protocol for this study was approved by the ethics committee of Yantai Yuhuangding Hospital, and written informed consent was obtained from each participant. Blood samples from 94 consecutive patients diagnosed with bladder cancer who underwent surgery at the Department of Urology, Yantai Yuhuangding Hospital from June 2007 to May 2013 were investigated. All the sixty control subjects were randomly selected among individuals receiving health examinations at the Health Examination Center of Yantai Yuhuangding Hospital, with subjects who had a history of cancer excluded from the study. Blood samples were collected from patients at the time of diagnosis prior to surgery. The demographic and pathological data, including age, gender, and the tumor stage, were obtained by a review of the patients' medical records. Venous blood samples were collected in anticoagulant-free tubes and centrifuged to obtain serum samples, which were stored at $-80^{\circ} \mathrm{C}$ until analysis. 


\section{Enzyme-linked immunosorbent assay (ELISA)}

Serum DKK-1 expression levels were measured by ELISA using an immunoassay kit (Miltenyi, Germany) according to the manufacturer's directions. The optical density (OD) at $450 \mathrm{~nm}$ was determined. The standard curves were established with OD450 as the Y-axis and the concentration of the standard substance as the $\mathrm{X}$-axis. The protein level was determined using a standard curve. The results are reported as the concentration of DKK-1 $(\mathrm{ng} / \mathrm{mL})$ in samples.

\section{Statistical analysis}

The expression levels of DKK-1 in serum were compared using the Student $t$-test or the Mann-Whitney U-test. The Kaplan-Meier method was used to estimate survival, and the log-rank test was used to test differences between the survival curves. Multivariate survival analysis was performed for all parameters significant in the univariate analyses using the Cox regression model. Results were considered statistically significant at $P<0.05$. All statistical analyses were performed using Statistical Package for Social Science (SPSS; version 18.0) for Windows (SPSS Inc, Chicago, IL).

\section{RESULTS}

\section{DKK-1 levels in the serum of patients with bladder cancer and their relationships with clinicopathological characteristics}

The mean serum level of DKK-1 in patients with bladder cancer was $35.91 \pm 16.09 \mathrm{ng} / \mathrm{mL}$, which was significantly higher than that in healthy individuals $(9.08 \pm 5.21 \mathrm{ng} / \mathrm{mL}, P<0.001$, Figure 1). The DKK-1 expression level was classified as high or low in relation to the median value, and patients with bladder cancer $(N=94)$ were divided into high $(N=48)$ and low $(N=46)$ expression groups. The relationships between serum DKK-1 levels and the clinicopathological characteristics of patients with bladder cancer were analyzed (Table 1). Serum DKK-1 levels were correlated significantly with lymph node metastasis $(P=0.021)$, distant metastasis $(P=0.013)$, and TNM stage $(P=0.008)$. However, there was no significant correlation of serum DKK-1 levels with age, gender, and tumor grade (all $P>0.05$ ).

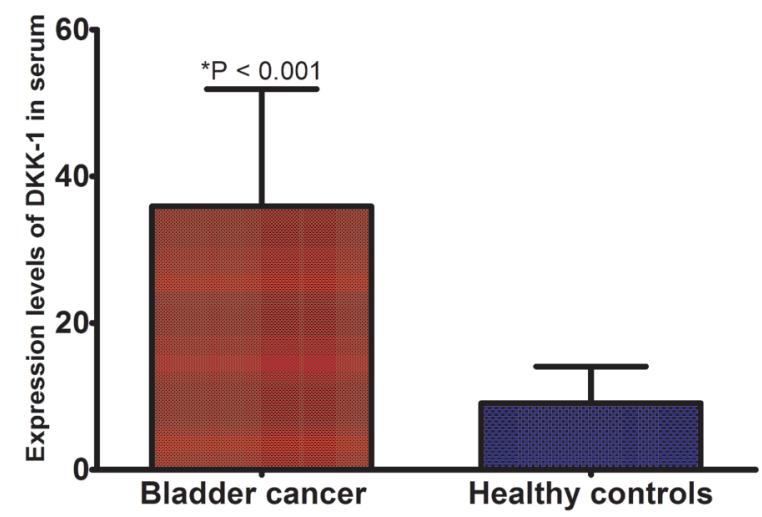

Figure 1. Mean serum level of Dickkopf-1 (DKK-1) significantly higher in patients with bladder cancer than in healthy individuals $(P<0.001)$. 
Table 1. Patient characteristics and clinicopathologic correlationswith serum Dickkopf-1 (DKK-1) expression levels.

\begin{tabular}{|c|c|c|c|c|}
\hline \multirow[t]{2}{*}{ Clinical variable } & \multirow[t]{2}{*}{ All cases } & \multicolumn{2}{|c|}{ Serum DKK-1 level } & \multirow[t]{2}{*}{$P$ value } \\
\hline & & High expression $(\mathrm{N}=48)$ & Low expression $(\mathrm{N}=46)$ & \\
\hline \multicolumn{5}{|l|}{ Age } \\
\hline$<60$ years & 39 & 17 & 22 & 0.365 \\
\hline$\geq 60$ years & 55 & 31 & 24 & \\
\hline \multicolumn{5}{|l|}{ Gender } \\
\hline Male & 56 & 27 & 29 & 0.582 \\
\hline Female & 38 & 21 & 17 & \\
\hline \multicolumn{5}{|c|}{ Lymph node metastases } \\
\hline No & 51 & 16 & 35 & 0.021 \\
\hline Yes & 43 & 32 & 11 & \\
\hline \multicolumn{5}{|c|}{ Distant metastases } \\
\hline No & 67 & 28 & 39 & 0.013 \\
\hline Yes & 27 & 20 & 7 & \\
\hline \multicolumn{5}{|l|}{ TNM stage } \\
\hline I-II & 52 & 17 & 35 & 0.008 \\
\hline III-IV & & 42 & 31 & 11 \\
\hline \multicolumn{5}{|l|}{ Grade } \\
\hline $\mathrm{G} 1 / 2$ & 61 & 29 & 32 & 0.076 \\
\hline G3 & 33 & 19 & 14 & \\
\hline
\end{tabular}

\section{Association of serum DKK-1 levels with prognosis in patients with bladder cancer}

Kaplan-Meier analysis with the log-rank test indicated that high serum DKK-1 levels were associated with significantly worse overall survival at 5 years $(33.4$ vs $70.1 \%, P=0.007$, Figure 2$)$. Univariate and multivariate analyses were utilized to evaluate whether the serum DKK-1 level and various clinicopathological features were independent prognostic parameters of patient outcomes. Multivariate analysis revealed that the serum DKK-1 level was an independent prognostic factors for overall survival [hazard ratio $(\mathrm{HR})=2.365,95 \%$ confidence interval $(\mathrm{Cl})=1.873-8.881, \mathrm{P}=$ 0.029 , Table 2] in patients with bladder cancer.

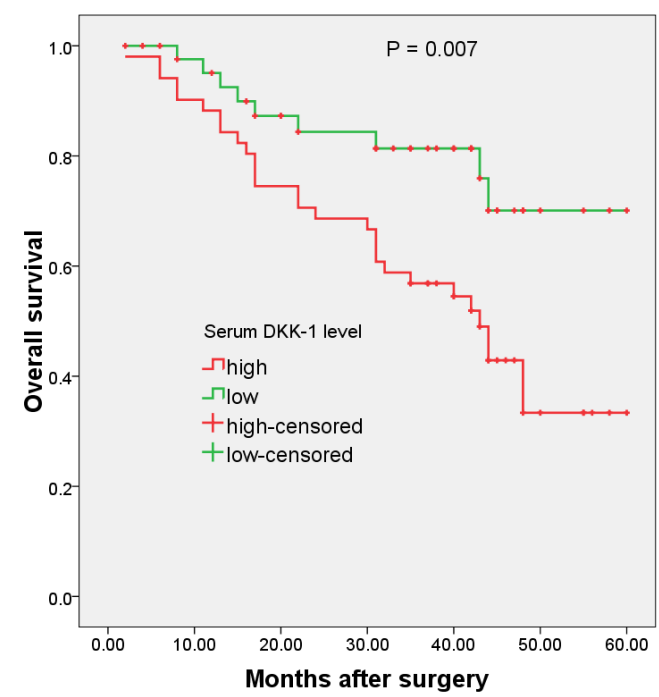

Figure 2. Kaplan-Meier analysis with the log-rank test indicating that high serum Dickkopf-1 (DKK-1) levels were associated with poorer overall survival $(P=0.007)$. 
Table 2. Summary of multivariate Cox regression analyses of overall survival.

\begin{tabular}{llll}
\hline Parameter & HR & \multicolumn{1}{c}{$95 \% \mathrm{Cl}$} & $\mathrm{P}$ value \\
\hline Gender & 0.871 & $0.278-2.881$ & 0.523 \\
Age & 1.762 & $0.675-3.187$ & 0.261 \\
TNM stage & 2.955 & $1.291-10.275$ & 0.005 \\
Lymph node metastases & 1.529 & $1.018-7.017$ & 0.012 \\
Distant metastasis & 4.526 & $3.162-11.923$ & 0.007 \\
Tumor grade & 1.924 & $0.892-9.023$ & 0.091 \\
Serum DKK-1 level & 2.365 & $1.873-8.881$ & 0.029 \\
\hline
\end{tabular}

Abbreviations: HR, hazard ratio; Cl, confidence interval; DKK-1, Dickkopf-1.

\section{DISCUSSION}

Currently, the outcome of bladder cancer is worse with tumor progression (Kaufman et al., 2009). Conventional clinicopathological factors are insufficient to predict the outcome of all patients with bladder cancer accurately. Therefore, new markers are needed to predict the course of bladder cancer (Galustian, 2013; Musquera et al., 2013). Further, the identification of such biomarkers is critical to refining our understanding of the pathogenesis of the disease, the biological basis for outcome disparities, and the development of more efficient treatment and surveillance strategies.

The Wnt pathway plays an important role in cancer (Polakis, 2000). Nineteen closely related Wnt genes have been identified in humans (Katoh and Katoh, 2007). The primary receptors of Wnt molecules are the seven-transmembrane Frizzled proteins (FRPs), each of which interacts with a single transmembrane low-density lipid (LDL) receptor-related protein 5/6 (LRP5/6) (He et al., 2004). Different secreted proteins, including soluble FRP-related proteins, Wnt inhibitory factor-1, and DKK-1-4 prevent ligand-receptor interactions and consequently inhibit the Wnt signaling pathway (Kawano and Kypta, 2003). Wnt proteins activate several intracellular signaling pathways through either a "canonical" or "non-canonical" pathway (Pandur et al., 2002). In the canonical pathway, secreted Wnt ligands bind to FRP proteins and regulate the stability of $\beta$-catenin (Nusse, 2005). In the absence of a Wnt signal, $\beta$-catenin is constitutively downregulated by a multicomponent destruction complex (Aberle et al., 1997). In the presence of secreted Wnt, this degradative process is inhibited, leading to the accumulation of $\beta$-catenin in the nucleus and regulation of Wnt-responsive genes (Eastman and Grosschedl, 1999). DKK-1 binds to LRP5 and blocks its interaction with Wnt-1, resulting in $\beta$-catenin degradation and effects on proliferation (Semenov et al., 2001; Mao et al., 2002). Another study illustrated that DKK-1 functions as both an antagonist of the $\mathrm{Wnt} / \beta$-catenin pathway and an agent that can upregulate other Wnt signaling pathways if the requisite Wnt/receptor combinations are available (Koppen et al., 2007). DKK-1 also can suppress cell growth and induce apoptotic cell death by activating the c-Jun N-terminal kinase pathway (Polakis, 2000). DKK-1 might promote cancer invasion through inducing filopodia extension and actin filament rearrangement by activating P-JNK1 (Wang and Zhang, 2011).

Previously, Kaba et al. (2014) investigated the clinical significance of serum DKK-1 levels in Turkish patients with bladder cancer, and the results of their study demonstrated that the level of serum DKK-1 was correlated with both disease progression and an increase in the tumor grade. However, the clinical significance and prognostic value of serum DKK-1 levels have not been previously investigated for bladder cancer in the Chinese population. In the present study, we investigated the clinical significance of DKK-1 in bladder cancer in the Chinese population. ELISA illustrated that the mean serum level of DKK-1 in patients with bladder cancer was significantly higher than that in healthy individuals. Furthermore, serum DKK-1 levels were correlated significantly 
with lymph node metastasis, distant metastasis, and TNM stage. These results demonstrated that increased serum levels of DKK-1 in patients with bladder cancer were associated with significantly aggressive pathologic features, indicating that serum DKK-1 levels might be associated with bladder cancer progression and poor prognosis. Kaplan-Meier analysis with the log-rank test indicated that high serum DKK-1 levels significantly affected overall survival. Multivariate analysis revealed that the serum DKK-1 level was an independent prognostic factor for overall survival $(\mathrm{HR}=2.365$, $95 \% \mathrm{Cl}=1.873-8.881, \mathrm{P}=0.029)$ in bladder cancer. Empirically, an HR of more than 1.5 is considered a strong prognostic factor. Therefore, serum DKK-1 levels might have considerable potential for predicting prognosis among patients with bladder cancer.

In conclusion, high serum DKK-1 levels may be associated with tumor progression and poor prognosis in bladder cancer and may be used as a potential biomarker to predict the prognosis of patients with bladder cancer.

\section{Conflicts of interest}

The authors declare no conflict of interest.

\section{REFERENCES}

Aberle H, Bauer A, Stappert J, Kispert A, et al. (1997). Beta-catenin is a target for the ubiquitin-proteasome pathway. EMBO J. 16: $3797-3804$.

Darlavoix T, Seelentag W, Yan P, Bachmann A, et al. (2009). Altered expression of CD44 and DKK1 in the progression of Barrett's esophagus to esophageal adenocarcinoma. Virchows Arch. 454: 629-637.

Eastman Q and Grosschedl R (1999). Regulation of LEF-1/TCF transcription factors by Wnt and other signals. Curr. Opin. Cell Biol. 11: 233-240

Forget MA, Turcotte S, Beauseigle D, Godin-Ethier J, et al. (2007). The Wnt pathway regulator DKK1 is preferentially expressed in hormone-resistant breast tumours and in some common cancer types. Br. J. Cancer 96: 646-653.

Galustian C. (2013). Tools to investigate biomarker expression in bladder cancer progression. BJU Int. 112: 404-406.

He X, Semenov M, Tamai K and Zeng X (2004). LDL receptor-related proteins 5 and 6 in Wnt/beta-catenin signaling: arrows point the way. Development 131: 1663-1677.

Kaba M, Pirincci N, Benli E, Gecit I, et al. (2014). Dickkopf-1 levels in Turkish patients with bladder cancer and its association with clinicopathological features. Asian Pac J. Cancer Prev. 15: 381-384.

Katoh M and Katoh M (2007). WNT signaling pathway and stem cell signaling network. Clin. Cancer Res. 13: 4042-4045.

Kaufman DS, Shipley WU, and Feldman S (2009). Bladder cancer. Lancet 374: 239-249.

Kawano Y and Kypta R (2003). Secreted antagonists of the Wnt signalling pathway. J. Cell Sci. 116: 2627-2634.

Koppen A, Ait-Aissa R, Hopman S, Koster J, et al. (2007). Dickkopf-1 is down-regulated by MYCN and inhibits neuroblastoma cell proliferation. Cancer Lett. 256: 218-228.

Makino T, Yamasaki M, Takemasa I, Takeno A, et al. (2009). Dickkopf-1 expression as a marker for predicting clinical outcome in esophageal squamous cell carcinoma. Ann. Surg. Oncol. 16: 2058-2064.

Mao B, Wu W, Davidson G, Marhold J, et al. (2002). Kremen proteins are Dickkopf receptors that regulate Wnt/beta-catenin signalling. Nature 417: 664-667.

Musquera M, Mengual L and Ribal MJ (2013). [Non-invasive diagnosis bladder cancer: new molecular markers and future perspectives]. Arch. Esp. Urol. 66: 487-494.

Nusse R. (2005). Wnt signaling in disease and in development. Cell Res. 15: 28-32.

Pandur P, Maurus D, and Kuhl M. (2002). Increasingly complex: new players enter the Wnt signaling network. BioEssays 24: 881-884.

Patil MA, Chua MS, Pan KH, Lin R, et al. (2005). An integrated data analysis approach to characterize genes highly expressed in hepatocellular carcinoma. Oncogene 24: 3737-3747.

Polakis P (2000). Wnt signaling and cancer. Genes Dev. 14, 1837-1851.

Ro JY, Staerkel GA and Ayala AG (1992). Cytologic and histologic features of superficial bladder cancer. Urol. Clin. North. Am. 19: 435-453. 
Semenov MV, Tamai K, Brott BK, Kuhl M, et al. (2001). Head inducer Dickkopf-1 is a ligand for Wnt coreceptor LRP6. Curr. Biol. 11: 951-961.

Siegel R, Ma J, Zou Z and Jemal A (2014). Cancer statistics, 2014. CA Cancer. J. Clin. 64: 9-29.

Wang $S$ and Zhang $S$ (2011). Dickkopf-1 is frequently overexpressed in ovarian serous carcinoma and involved in tumor invasion. Clin. Exp. Metastasis 28: 581-591.

Yamabuki T, Takano A, Hayama S, Ishikawa N, et al. (2007). Dikkopf-1 as a novel serologic and prognostic biomarker for lung and esophageal carcinomas. Cancer Res. 67: 2517-2525. 